Journal of Mathematics and Statistics 4 (2): 70-74, 2008

ISSN 1549-3644

(C) 2008 Science Publications

\title{
Weyl's Type Theorems for Quasi-Class A Operators
}

\author{
M.H.M. Rashid, M.S.M. Noorani and A.S. Saari \\ School of Mathematical Sciences, Faculty of Science and \\ Technology, Universiti Kebangsaan Malaysia, 43600 UKM, Selangor \\ Darul Ehsan, Malaysia
}

\begin{abstract}
A variant of Weyl theorem for a class of quasi-class A acting on an infinite complex Hilbert space were discussed. If the adjoint of $\mathrm{T}$ is a quasi-class $\mathrm{A}$ operator, then the generalized a-Weyl holds for $\mathrm{f}(\mathrm{T})$, for every function that analytic on the spectrum of $\mathrm{T}$. The generalized Weyl theorem holds for a quasi-class A was proved. Also, a characterization of the Hilbert space as a direct sum of range and kernel of a quasi-class A was given. Among other things, if the operator is a quasi-class A, then the B-Weyl spectrum satisfies the spectral theorem was characterized.
\end{abstract}

Key words: Single valued Extension property, Fredholm theory, Browder's spectrum theory

\section{INTRODUCTION}

Throughout this study let $\mathrm{B}(\mathrm{H})$ and $\mathrm{K}(\mathrm{H})$, denote, respectively, the algebra of bounded linear operators and the ideal of compact operators acting on an infinite dimensional separable Hilbert space $H$. If $T \in B(H)$ we shall write $\operatorname{ker}(\mathrm{T})$ and $\operatorname{ran}(\mathrm{T})$ for the null space and range of $\mathrm{T}$, respectively. Also, let $\alpha(\mathrm{T}):=\operatorname{dim} \operatorname{ker}(\mathrm{T})$, $\beta(\mathrm{T}):=$ co $\operatorname{dim} \operatorname{ran}(\mathrm{T})$ and let $\sigma(\mathrm{T}), \sigma_{\mathrm{a}}(\mathrm{T}) \sigma_{\mathrm{p}}(\mathrm{T})$ denote the spectrum, approximate point spectrum and point spectrum of $\mathrm{T}$, respectively. An operator $T \in B(H)$ is called Fredholm if it has closed range, finite dimensional null space and its range has finite co dimension. The index of a Fredholm operator is given by

$$
\mathrm{i}(\mathrm{T}):=\alpha(\mathrm{T})-\beta(\mathrm{T})
$$

$\mathrm{T}$ is called Weyl if it is Fredholm of index 0 and Browder if it is Fredholm of finite ascent and descent.

The essential spectrum $\sigma_{\mathrm{e}}(\mathrm{T})$, the Weyl spectrum $\sigma_{\mathrm{W}}(\mathrm{T})$ and the Browder spectrum $\sigma_{\mathrm{b}}(\mathrm{T})$ of $\mathrm{T}$ are defined by

$$
\begin{gathered}
\sigma_{\mathrm{e}}(\mathrm{T})=\{\lambda \in \mathrm{C}: \mathrm{T}-\lambda \mathrm{I} \text { is not Fredholm }\}, \\
\sigma_{\mathrm{W}}(\mathrm{T})=\{\lambda \in \mathrm{C}: \mathrm{T}-\lambda \mathrm{I} \text { is not Weyl }\},
\end{gathered}
$$

and

$$
\sigma_{b}(T)=\{\lambda \in C: T-\lambda I \text { is not Browder }\},
$$

$$
\sigma_{\mathrm{e}}(\mathrm{T}) \subseteq \sigma_{\mathrm{W}}(\mathrm{T}) \subseteq \sigma_{\mathrm{b}}(\mathrm{T}) \subseteq \sigma_{\mathrm{e}}(\mathrm{T}) \cup \operatorname{acc} \sigma(\mathrm{T})
$$

Where, we write accK for the accumulation points of $\mathrm{K} \subseteq \mathrm{C}$. If we write isoK $=\mathrm{K}-\operatorname{accK}$ then we let

$$
\mathrm{E}_{0}(\mathrm{~T}):=\{\lambda \in \operatorname{iso\sigma }(\mathrm{T}): 0<\alpha(\mathrm{T}-\lambda \mathrm{I})<\infty\}
$$

for the isolated eigenvalues of finite multiplicity and

$$
\mathrm{p}_{0}(\mathrm{~T}):=\sigma(\mathrm{T})-\sigma_{\mathrm{b}}(\mathrm{T})
$$

for the Riesz points of T. Then (1.1) with the help of "Punctured neighborhoods Theorem"

$$
\operatorname{iso\sigma }(\mathrm{T})-\sigma_{\mathrm{e}}(\mathrm{T})=\operatorname{i\operatorname {so\sigma }}(\mathrm{T})-\sigma_{\mathrm{W}}(\mathrm{T})=\mathrm{p}_{0}(\mathrm{~T}) \subseteq \mathrm{E}_{0}(\mathrm{~T})
$$

Definition 1: ${ }^{[6]}$ We say that Weyl's theorem holds for $\mathrm{T} \in \mathrm{B}(\mathrm{H})$ if

$$
\sigma(\mathrm{T})-\sigma_{\mathrm{W}}(\mathrm{T})=\mathrm{E}_{0}(\mathrm{~T})
$$

and we shall say that Browder's theorem holds for $\mathrm{T} \in \mathrm{B}(\mathrm{H})$ if

$$
\sigma(\mathrm{T})-\sigma_{\mathrm{W}}(\mathrm{T})=\mathrm{p}_{0}(\mathrm{~T})
$$

Evidently Weyl's theorem implies Browder's theorem. Let us denote by:

$$
\Phi_{+}(\mathrm{H})=\{\mathrm{T} \in \mathrm{B}(\mathrm{H}): \alpha(\mathrm{T})<\infty \text { and } \operatorname{ran}(\mathrm{T}) \text { is closed }\}
$$

Corresponding Author: M.H.M. Rashid, School of Mathematical Sciences, Faculty of Science and Technology, Universiti Kebangsaan Malaysia, 43600 UKM, Selangor Darul Ehsan, Malaysia 
the class of all upper semi-Fredholm operators and

$$
\Phi_{-}(\mathrm{H})=\{\mathrm{T} \in \mathrm{B}(\mathrm{H}): \beta(\mathrm{T})<\infty\}
$$

the class of all lower semi-Fredholm operators. The class of all semi-Fredholm operators is defined by $\Phi_{ \pm}(\mathrm{H})=\Phi_{+}(\mathrm{H}) \cup \Phi_{-}(\mathrm{H})$, whilst the class of all Fredholm operators is defined by $\Phi(H)=\Phi_{+}(H) \cap \Phi_{-}(H)$. The ascent $\mathrm{a}:=\mathrm{a}(\mathrm{T})$ of an operator $\mathrm{T}$ is the smallest non-negative integer s such that $\operatorname{ker}\left(\mathrm{T}^{\mathrm{s}}\right)=\operatorname{ker}\left(\mathrm{T}^{\mathrm{s}+1}\right)$. If such integer does not exist we put $\mathrm{a}(\mathrm{T})=\infty$.

Analogously, the descent $d=d(T)$ of an operator $\mathrm{T}$ is the smallest non-negative integer $t$ such that $\operatorname{ran}\left(\mathrm{T}^{\mathrm{t}}\right)$ $=\operatorname{ran}\left(T^{t+1}\right)$ and if such integer does not exist we put $\mathrm{d}(\mathrm{T})=\infty$. It is well-known that if $\mathrm{a}(\mathrm{T})$ and $\mathrm{d}(\mathrm{T})$ are both finite then $\mathrm{a}(\mathrm{T})=\mathrm{d}(\mathrm{T})$ [7,proposition 1.49]. Two other important classes of operators in Fredholm theory are the class of all upper semi-Browder operators

$$
\mathrm{B}_{+}(\mathrm{H}):=\left\{\mathrm{T} \in \Phi_{+}(\mathrm{H}): \mathrm{a}(\mathrm{T})<\infty\right\}
$$

and the class of all lower semi-Browder operators

$$
B_{-}(H):=\left\{T \in \Phi_{-}(H): d(T)<\infty\right\} \text {. }
$$

The class of all Browder operators is defined by $\operatorname{Bro}(H):=B_{+}(H) \cap B_{-}(H)$. Note that if $\mathrm{T} \in \mathrm{B}_{+}(\mathrm{H})$ then the index is defined by $\mathrm{i}(\mathrm{T})=\alpha(\mathrm{T})-\beta(\mathrm{T})$ is less than or equal to 0 , whilst if $\mathrm{T} \in \mathrm{B}_{-}(\mathrm{H})$, then $\mathrm{i}(\mathrm{T}) \geq 0,{ }^{[14]}$. The class of all Weyl Operators $\mathrm{W}(\mathrm{H})$ is defined by

$$
\mathrm{W}(\mathrm{H})=\{\mathrm{T} \in \Phi(\mathrm{H}): \mathrm{i}(\mathrm{T})=0\} .
$$

Note that $\mathrm{Bro}(\mathrm{H}) \subseteq \mathrm{W}(\mathrm{H})$, since every Fredholm operator with finite ascent and finite descent has necessary index $0,{ }^{[1,9,10]}$. The classes of operators defined above motivate the definition of several spectra. The essential approximate point spectrum is

$$
\sigma_{\text {ea }}(T):=\bigcap\left\{\sigma_{\mathrm{a}}(\mathrm{T}+\mathrm{K}): \mathrm{K} \in \mathrm{K}(\mathrm{H})\right\} \text {, }
$$

and

$$
\sigma_{\mathrm{ba}}(\mathrm{T}):=\bigcap\left\{\sigma_{\mathrm{a}}(\mathrm{T}+\mathrm{K}): \mathrm{TK}=\mathrm{KT}, \mathrm{K} \in \mathrm{K}(\mathrm{H})\right\},
$$

is the Browder essential approximate point spectrum. It is well-known that $\sigma_{\text {ea }}(T)=\left\{\lambda \in \mathrm{C}: T-\lambda \mathrm{I} \notin \mathrm{B}_{+}(\mathrm{H})\right\}$.

Definition 2: ${ }^{[1]}$ We say that a-Browder's holds for $\mathrm{T}$ if

$$
\sigma_{\mathrm{ea}}(\mathrm{T})=\sigma_{\mathrm{ba}}(\mathrm{T}) \text {. }
$$

It is known that if $\mathrm{T} \in \mathrm{B}(\mathrm{H})$ then a-Browder's theorem implies Browder's theorem. In ${ }^{[8]}$, the authors proved that Weyl's theorem holds for quasi-classA, in this paper, we prove that generalized Weyl's holds for quasi-class A operators.

\section{RESULTS}

Definition 3: An operator $T \in B(H)$ is said to be quasiclass $\mathrm{A}$ if

$$
\mathrm{T}^{*}\left|\mathrm{~T}^{2}\right| \mathrm{T} \geq \mathrm{T}^{*}|\mathrm{~T}|^{2} \mathrm{~T} \text {. }
$$

The class of quasi-class A introduced and studied by Jeon and Kim ${ }^{[15]}$, for more interesting properties the reader should refer to ${ }^{[8,15]}$.

Lemma 4: Let $\mathrm{T} \in \mathrm{B}(\mathrm{H})$ be a quasi-class $\mathrm{A}$. Then $\mathrm{H}=\operatorname{ran}(\mathrm{T}) \oplus \operatorname{ker}(\mathrm{T})$. Moreover $\mathrm{T}_{1}$, the restriction of $\mathrm{T}$ to $\operatorname{ran}(\mathrm{T})$ is one-one and onto.

Proof: Suppose that

$$
\begin{aligned}
& y \in \operatorname{ran}(T) \cap \operatorname{ker}(T) \text { then } y=T x \\
& \text { for some } x \in H \text { and } T y=0 .
\end{aligned}
$$

It follows that $\mathrm{T}^{2} \mathrm{x}=0$ However, $\mathrm{a}(\mathrm{T})=1$ and so $\mathrm{x} \in \operatorname{ker}\left(\mathrm{T}^{2}\right)=\operatorname{ker}(\mathrm{T})$. Hence $\mathrm{y}=\mathrm{Tx}=0 \quad$ and $\quad$ so $\operatorname{ran}(T) \cap \operatorname{ker}(T)=\{0\} . \quad$ Also, $\quad T(\operatorname{ran}(T))=\operatorname{ran}(T)$. If $x \in H$ there is $u \in \operatorname{ran}(T)$ such that

$\mathrm{Tu}=\mathrm{Tx}$. Now if $\mathrm{z}=\mathrm{x}-\mathrm{u}$ then $\mathrm{T}_{\mathrm{z}}=0$ Hence $H=\operatorname{ran}(T) \oplus \operatorname{ker}(T)$. Since $d(T)=1, T$ maps $\operatorname{ran}(T)$ onto itself. If $y \in \operatorname{ran}(T)$ and $T y=0$ then $\mathrm{y} \in \operatorname{ran}(\mathrm{T}) \cap \operatorname{ker}(\mathrm{T})=\{0\}$. Hence $\mathrm{T}_{1}$ is one-one and onto.

Recall that an operator $\mathrm{S} \in \mathrm{B}(\mathrm{H})$ is said to be quasiaffine transform of $\mathrm{T}$ (abbreviate $\mathrm{S} \prec \mathrm{T}$ ) if there is a quasiaffinity $\mathrm{X}$ such that $\mathrm{XS}=\mathrm{TX}$.

Definition 5: ${ }^{[13]}$ Let $\operatorname{Hol}(\sigma(\mathrm{T}))$ be the space of all functions that analytic in an open neighborhoods of $\sigma(T)$. We say that $T \in B(H)$ has the single-valued extension property (SVEP) if for every open set $\mathrm{U} \subseteq \square$ the only analytic function $\mathrm{f}: \mathrm{U} \rightarrow \mathrm{H}$ which satisfies the equation $(T-\lambda I) f(\lambda)=0$ is the constant function $\mathrm{f}=0$.

It is well-known that $\mathrm{T} \in \mathrm{B}(\mathrm{H})$ has SVEP at every point of the resolvent $\rho(T):=\square-\sigma(T)$. Moreover, from the identity theorem for analytic function it easily follows that $\mathrm{T} \in \mathrm{B}(\mathrm{H})$ has SVEP at every point of the boundary $\partial \sigma(T)$ of the spectrum. In particular, $T$ has SVEP at every isolated point of $\sigma(\mathrm{T}){ }^{[16]}$. In ${ }^{[18, \text { proposition }}$ ${ }^{1.8]}$, Laursen proved that if $\mathrm{T}$ is of finite ascent, then $\mathrm{T}$ has SVEP. 
Lemma 6: If $\mathrm{T} \in \mathrm{B}(\mathrm{H})$ is a quasi-class $\mathrm{A}$ operator and $S \prec T$. Then $S$ has SVEP.

Proof: Since $\mathrm{T}$ is a quasi-class A operator and it has a SVEP, then the result follows from ${ }^{[6]}$.

For $\mathrm{T} \in \mathrm{B}(\mathrm{H})$, it is known that the inclusion $\sigma_{\text {ea }}(\mathrm{f}(\mathrm{T})) \subseteq \mathrm{f}\left(\sigma_{\text {ea }}(\mathrm{T})\right)$ holds for every $\mathrm{f} \in \operatorname{Hol}(\sigma(\mathrm{T}))$, with no restriction on $\mathrm{T}$. The next theorem shows that for quasi-class $\mathrm{A}$ operators the spectral mapping theorem holds for the essential approximate point spectrum.

Theorem 7: If $\mathrm{T} \in \mathrm{B}(\mathrm{H})$ is a quasi-class $\mathrm{A}$ operator. Then $\quad \sigma_{\text {ea }}(\mathrm{f}(\mathrm{T}))=\mathrm{f}\left(\sigma_{\mathrm{ea}}(\mathrm{T})\right)$ holds for every $f \in \operatorname{Hol}(\sigma(\mathrm{T}))$.

Proof. Let $f \in \operatorname{Hol}(\sigma(\mathrm{T}))$. It suffices to show that $\sigma_{\text {ea }}(\mathrm{f}(\mathrm{T})) \supseteq \mathrm{f}\left(\sigma_{\mathrm{ea}}(\mathrm{T})\right)$. Suppose that $\lambda \notin \sigma_{\mathrm{ea}}(\mathrm{f}(\mathrm{T}))$ then $\mathrm{f}(\mathrm{T})-\lambda \mathrm{I} \in \Phi_{+}(\mathrm{H}) \quad$ and $\quad \mathrm{i}(\mathrm{f}(\mathrm{T})-\lambda \mathrm{I}) \leq 0 \quad$ and $\mathrm{f}(\mathrm{T})-\lambda \mathrm{I}=\mathrm{c}\left(\mathrm{T}-\alpha_{1} \mathrm{I}\right) \ldots\left(\mathrm{T}-\alpha_{\mathrm{n}}\right) \mathrm{g}(\mathrm{T}), \quad$ Where, $\mathrm{c}, \alpha_{1}, \ldots, \alpha_{\mathrm{n}} \in \square$ and $\mathrm{g}(\mathrm{T})$ is invertible. If $\mathrm{T}$ is a quasiclass $A$, then $\sum_{j=1}^{n} i\left(T-\alpha_{j} I\right) \leq 0$ and $i\left(T-\alpha_{j} I\right) \leq 0$ for each $j=1, \ldots, n$. Therefore $\lambda \notin f\left(\sigma_{\text {ea }}(T)\right)$. This completes the proof.

Definition 8: ${ }^{[12]}$ For $\mathrm{T} \in \mathrm{B}(\mathrm{H})$ and closed subset $\mathrm{F}$ of $\square$ the glocal spectral is

$$
\begin{aligned}
& { }_{\mathrm{T}}(\mathrm{F})=\{\mathrm{x} \in \mathrm{H}: \exists \text { analytic function } \mathrm{f}: \square-\mathrm{F} \rightarrow \mathrm{H} \\
& \text { such that }(\lambda \mathrm{I}-\mathrm{T}) \mathrm{f}(\lambda)=\mathrm{x}, \forall \mathrm{x} \in \square-\mathrm{F}\} .
\end{aligned}
$$

Definition 9: ${ }^{[12]}$ The quasinilpotent part $\mathrm{H}_{0}(\mathrm{~T}-\lambda \mathrm{I})$ and the analytic core $\mathrm{K}(\mathrm{T}-\lambda \mathrm{I})$ are defined by $H_{0}(T-\lambda I):=\left\{x \in H: \lim _{n \rightarrow \infty}\left\|(T-\lambda I)^{n} x\right\|^{\frac{1}{n}}=0\right\}$, and

$\mathrm{K}(\mathrm{T}-\lambda \mathrm{I})=\{\mathrm{x} \in \mathrm{H}$ : there exists a sequence $\left\{\mathrm{x}_{\mathrm{n}}\right\} \subset \mathrm{H}$ and $\delta>0$ for which $\mathrm{x}=\mathrm{x}_{0},(\mathrm{~T}-\lambda \mathrm{I}) \mathrm{x}_{\mathrm{n}+1}$

$=x_{n}$ and $\left\|x_{n}\right\| \leq \delta^{n}\|x\|$ for all $\left.n=1,2, \ldots\right\}$,

respectively.

Note that $\mathrm{H}_{0}(\mathrm{~T}-\lambda \mathrm{I})$ and $\mathrm{K}(\mathrm{T}-\lambda \mathrm{I})$ are generally non-closed hyper-invariant subspaces of $\mathrm{T}-\lambda \mathrm{I}$ such that

$(\mathrm{T}-\lambda \mathrm{I})^{-\mathrm{p}}(0) \subseteq \mathrm{H}_{0}(\mathrm{~T}-\lambda \mathrm{I})$ for all $\mathrm{p}=0,1,2, \ldots$ and
$(T-\lambda I) K(T-\lambda I)=K(T-\lambda I)$. For more information about this subject the reader should refer to ${ }^{[11,12]}$.

Recall that generalized Weyl's theorem (g-Weyl's) holds for $\mathrm{T}$ if $\sigma(\mathrm{T})-\sigma_{\mathrm{BW}}(\mathrm{T})=\mathrm{E}(\mathrm{T}), \quad$ Where, $\mathrm{E}(\mathrm{T})$ denotes the isolated points $\lambda$ of $\sigma(\mathrm{T})$, which are eigenvalues (no restriction on multiplicity) and $\sigma_{\mathrm{BW}}(\mathrm{T})$ is the set of all complex numbers $\lambda$ for which

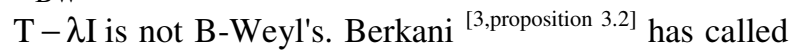
an operator $\mathrm{T} \in \mathrm{B}(\mathrm{H})$ is $\mathrm{B}$-Fredholm if there exists a natural number $\mathrm{n}$ for which the induced operator $T_{n}: \operatorname{ran}\left(T^{n}\right) \rightarrow \operatorname{ran}\left(T^{n}\right)$ is Fredholm in the usual sense and B-Weyl's" if in addition $\mathrm{T}_{\mathrm{n}}$ has zero index. Berkani [3,corollary ${ }^{3.3]}$ has shown that, if $\mathrm{g}$-Weyl's theorem holds for $\mathrm{T}$ then so does Weyl's theorem.

For the sake of simplicity of notation we introduce the abbreviations $\mathrm{gaW}, \mathrm{aW}, \mathrm{gW}$ and $\mathrm{W}$ to signify that an operator $\mathrm{T} \in \mathrm{B}(\mathrm{H})$ (which is usually understood) obeys generalized a-Weyl's theorem, a- Weyl's theorem, generalized Weyl's theorem and Weyl's theorem, respectively.

Analogous meaning is attached to the abbreviations $\mathrm{gaB}, \mathrm{aB}, \mathrm{gB}$ and $\mathrm{B}$ with respect to Browder's theorem. In the following diagrams, arrows signify implications between various Weyl's and Browder's theorems ${ }^{[2,4,5,20]}$.

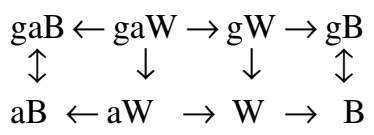

Theorem 10: If $\mathrm{T} \in \mathrm{B}(\mathrm{H})$ is a quasi-class $\mathrm{A}$ operator. Then $\quad \sigma_{\mathrm{BW}}(\mathrm{f}(\mathrm{T}))=\mathrm{f}\left(\sigma_{\mathrm{BW}}(\mathrm{T})\right)$ holds for every $f \in \operatorname{Hol}(\sigma(\mathrm{T}))$.

Proof: It is suffices to show $\sigma_{\mathrm{BW}}(\mathrm{f}(\mathrm{T})) \supseteq \mathrm{f}\left(\sigma_{\mathrm{BW}}(\mathrm{T})\right)$ since the other inclusion holds for every $\mathrm{f} \in \operatorname{Hol}(\sigma(\mathrm{T}))$ with no restriction on $\mathrm{T}$. Let $\mu \in \sigma_{\mathrm{BW}}(\mathrm{T})$, and $\mathrm{f} \in \operatorname{Hol}(\sigma(\mathrm{T}))$. Since $\sigma(\mathrm{T})$ is a compact subset of $\square$, the function $f(z)-f(\mu)$ possesses at most a finite number of zeros in $\sigma(T)$. So

$$
f(T)-f(\mu I)=(T-\mu I) \prod_{j=1}^{m}\left(T-\lambda_{j} I\right) g(T),
$$

Where, $\mu, \lambda_{1}, \ldots, \lambda_{\mathrm{n}} \in \square$ and $\mathrm{g}(\mathrm{T})$ is an invertible operator. So $\mathrm{g}(\mathrm{T})^{-1}$ is a B-Weyl's operator. If $\mathrm{f}(\mathrm{T})-\mathrm{f}(\mu \mathrm{I})$ is B-Weyl's operator, by ${ }^{[3]}$ applied to $\mathrm{f}(\mathrm{T})-\mathrm{f}(\mu \mathrm{I})$ and $\mathrm{g}(\mathrm{T})^{-1}$ we have 


$$
(\mathrm{f}(\mathrm{T})-\mathrm{f}(\mu \mathrm{I})) \mathrm{g}(\mathrm{T})^{-1}=(\mathrm{T}-\mu \mathrm{I})^{\mathrm{m}} \prod_{\mathrm{j}=1}^{\mathrm{n}}\left(\mathrm{T}-\lambda_{\mathrm{j}} \mathrm{I}\right)
$$

is a B-Weyl's operator. So from ${ }^{[3]} \mathrm{T}-\mu \mathrm{I}$ is B-Weyl's, a fact which contradicts our assumption. Hence $\mathrm{f}(\mu) \in \sigma_{\mathrm{BW}}(\mathrm{f}(\mathrm{T}))$ and $\mathrm{f}\left(\sigma_{\mathrm{BW}}(\mathrm{T})\right) \subseteq \sigma_{\mathrm{BW}}(\mathrm{f}(\mathrm{T}))$.

Theorem 11: Let $T$ be a quasi-class $A$ operator. Then generalized Weyl's theorem holds for $f(T)$ for all $\mathrm{f} \in \operatorname{Hol}(\sigma(\mathrm{T}))$.

Proof: Since $T$ is isoloid in $\sigma(T)$ by ${ }^{[8, \text { lemma } 1.8]}$ and has SVEP, then it suffices to prove that generalized Weyl's theorem holds for $\mathrm{T}$. We shall show that $\sigma(\mathrm{T})-\sigma_{\mathrm{BW}}(\mathrm{T})=\mathrm{E}(\mathrm{T}) . \quad$ Let $\lambda \in \sigma(\mathrm{T})-\sigma_{\mathrm{BW}}(\mathrm{T})$,

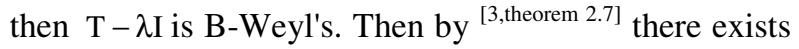
two closed subspaces $\mathrm{N}$ and $\mathrm{M}$ of $\mathrm{H}$ such that $\mathrm{H}=\mathrm{M} \oplus \mathrm{N}, \quad \mathrm{T}_{1}=(\mathrm{T}-\lambda \mathrm{I}) \mathrm{I}_{\mathrm{M}}$ is Weyl's operator, $\mathrm{T}_{2}=\left.(\mathrm{T}-\lambda \mathrm{I})\right|_{\mathrm{N}}$ is nilpotent and $\mathrm{T}-\lambda \mathrm{I}=\mathrm{T}_{1} \oplus \mathrm{T}_{2}$. We have two possibilities: either $\lambda \in \sigma\left(\mathrm{T} \mathrm{I}_{\mathrm{M}}\right)$ or $\lambda \notin \sigma\left(\mathrm{T} \mathrm{I}_{\mathrm{M}}\right)$.

Case I: $\lambda \in \sigma\left(\left.T\right|_{M}\right)$. Since $\left.T\right|_{M}$ is quasi-class $A$, then Weyl's theorem holds for $\mathrm{Tl}_{\mathrm{M}}$ and so if $\lambda \in \sigma\left(\mathrm{T} \mathrm{I}_{\mathrm{M}}\right)$, then $\quad \lambda \in \mathrm{E}_{0}\left(\mathrm{Tl}_{\mathrm{M}}\right) \subset \operatorname{iso\sigma }\left(\mathrm{T} \mathrm{I}_{\mathrm{M}}\right)$. Since $\mathrm{T}-\lambda \mathrm{I}=\left(\left.\mathrm{T}\right|_{\mathrm{M}}-\lambda \mathrm{I}_{\mathrm{M}}\right) \oplus \mathrm{T}_{2}$ and $\mathrm{T}_{2}$ is nilpotent, $\sigma\left(\mathrm{T}_{1}\right)-\{0\}=\sigma(\mathrm{T}-\lambda \mathrm{I})-\{0\} \quad$ and $\quad \lambda \in \operatorname{iso} \sigma(\mathrm{T})$. This implies that $\lambda \in \mathrm{E}_{0}(\mathrm{~T}) \subset \mathrm{E}(\mathrm{T})$.

Case II: $\lambda \notin \sigma\left(\left.T\right|_{M}\right)$. Then $\lambda$ is a pole of $T$ which implies that $\lambda \in \mathrm{E}(\mathrm{T})$. Conversely, let $\lambda \in \mathrm{E}(\mathrm{T})$. Let $\mathrm{P}$ be the spectral projection associated with $\lambda$, then $\operatorname{ran}(\mathrm{P})=\mathrm{H}_{0}(\mathrm{~T}-\lambda \mathrm{I}), \operatorname{ker}(\mathrm{P})=\mathrm{K}(\mathrm{T}-\lambda \mathrm{I})$,

$$
\begin{aligned}
& \mathrm{H}_{0}(\mathrm{~T}-\lambda \mathrm{I}) \neq 0, \mathrm{H}=\mathrm{H}_{0}(\mathrm{~T}-\lambda \mathrm{I}) \oplus \mathrm{K}(\mathrm{T}-\lambda \mathrm{I}), \\
& \mathrm{K}(\mathrm{T}-\lambda \mathrm{I}) \text { is } \quad \text { closed } \quad \text { subspace }{ }^{[16,19]}, \quad \text { Since } \\
& 0 \neq \operatorname{ker}(\mathrm{T}-\lambda \mathrm{I}) \subset \mathrm{H}_{0}(\mathrm{~T}-\lambda \mathrm{I}), \quad \lambda \quad \text { is a pole of the }
\end{aligned}
$$
resolvent $\Re_{\lambda}(T)=(T-\lambda I)^{-1}$, then by ${ }^{[16]}$ there is some $\mathrm{q}>0$ such that the space $(\mathrm{T}-\lambda \mathrm{I})^{-\mathrm{q}}(0)$ is non-zero and complemented by a closed T-invariant subspace $\operatorname{ran}\left((\mathrm{T}-\lambda \mathrm{I})^{\mathrm{q}}\right) \subset \operatorname{ran}(\mathrm{T}-\lambda \mathrm{I})$. Hence $\mathrm{T}-\lambda \mathrm{I}$ is $\mathrm{B}$-Weyl's, i.e., $\lambda \notin \sigma_{\mathrm{BW}}(\mathrm{T})$.

A bounded linear operator $\mathrm{T}$ is called a-isoloid if every isolated point of $\sigma_{a}(T)$ is an eigenvalue of $T$.

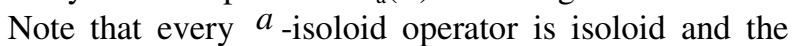
converse is not true in general.

Theorem 2.4 of ${ }^{[21]}$ affirms that if $\mathrm{T}^{*}$ or $\mathrm{T}$ has the SVEP and if $\mathrm{T}$ is a-isoloid and generalized a-Weyl's holds for $T$ then generalized a-Weyl's theorem holds for $\mathrm{f}(\mathrm{T})$, for every $f \in \operatorname{Hol}(\sigma(T))$. If $T^{*}$ is quasi-class $A$, then we have:

Theorem 12: Let $T^{*}$ be a quasi-class $A$ operator. Then generalized a-Weyl's theorem hold for T.

Proof: Since $T^{*}$ has SVEP then $\sigma(T)=\sigma_{a}(T)$ and consequently $\mathrm{E}(\mathrm{T})=\mathrm{E}^{\mathrm{a}}(\mathrm{T})$. Let $\lambda \notin \sigma_{\mathrm{SBF}_{+}^{-}}(\mathrm{T})$ be given, then $\mathrm{T}-\lambda \mathrm{I}$ is semi-B-Fredholm and $i(T-\lambda I) \leq 0$. Then [17,proposition 1.2] implies that $\mathrm{i}(\mathrm{T}-\lambda \mathrm{I})=0$ and consequently $\mathrm{T}-\lambda \mathrm{I}$ is $\mathrm{B}$-Weyl's. Hence $\lambda \notin \sigma_{\mathrm{BW}}(\mathrm{T})$. So it follows from ${ }^{[21 \text {,theorem } 3.1]}$ that $\lambda \in \mathrm{E}(\mathrm{T})=\mathrm{E}^{\mathrm{a}}(\mathrm{T})$. For the converse, let $\lambda \in \mathrm{E}^{\mathrm{a}}(\mathrm{T})$. Then $\lambda \in \operatorname{iso\sigma }_{\mathrm{a}}(\mathrm{T})$. Since $\mathrm{T}^{*}$ has the SVEP, we have $\sigma(T)=\sigma_{a}(T)$. Hence $\bar{\lambda} \in \sigma\left(T^{*}\right)$. Now we represent $\mathrm{T}^{*}$ as the direct sum $\mathrm{T}^{*}=\mathrm{T}_{1} \oplus \mathrm{T}_{2}$, Where, $\sigma\left(\mathrm{T}_{1}\right)=\{\bar{\lambda}\}$ and $\sigma\left(\mathrm{T}_{2}\right)=\sigma\left(\mathrm{T}_{1}\right)-\{\bar{\lambda}\}$. Since $\mathrm{T}$ is quasiclass $\mathrm{A}$ then so does $\mathrm{T}_{1}$ and so we have two cases:

Case I: $(\bar{\lambda}=0)$ : Then $T_{1}$ is quasinilpotent. Hence it follows that $T_{1}$ is nilpotent. Since $T_{2}$ is invertible, then $\mathrm{T}^{*}$ is a $B$-Weyl's.

Case II: $(\bar{\lambda} \neq 0)$ : Since $\sigma\left(T_{1}\right)=\{\bar{\lambda}\}$, then $T_{1}-\bar{\lambda}$ is nilpotent and $T_{2}-\bar{\lambda} I$ is invertible. Hence it follows

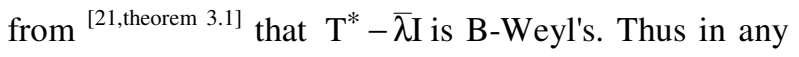
case $\lambda \in \sigma_{\mathrm{a}}(\mathrm{T})-\sigma_{\mathrm{SBF}_{+}^{-}}(\mathrm{T})$.

Theorem 13: Let $T \in B(H)$ and $T$ or $T^{*}$ is a quasiclass $\mathrm{A}$. Then the generalized a-Browder's theorem holds for $\mathrm{T}$.

Proof: The proof is a consequence immediate of ${ }^{[8,2]}$.

\section{CONCLUSION}

It can be shown that if $\mathrm{T}^{*}$ is a quasi-class $\mathrm{A}$ then the generalized a-Browder's theorem holds for $f(T)$ for every $\mathrm{f} \in \operatorname{Hol}(\sigma(\mathrm{T}))$. 


\section{REFERENCES}

1. Aiena, P. and P. Carpintero, 2005. Weyl's Theorem, a-Weyl's Theorem and Single-Valued Extension Property, Extracta Math., 20 (1): 25-41.

2. Amouch, M. and H. Zguitti, 2006. On the equivalence of Browder's and generalized Browder's theorem, Glasgow Math. J. 48: 179-185.

3. Berkani, M., 1999. On A class of Quasi-Fredholm Operators, Integ. Equat. Oper. Th., 34: 244-249.

4. Berkani, M., 2002. B-Weyl Spectrum and Poles of the Resolvent. Math. Anal. Appl., 272: 596-603.

5. Berkani, M. and J. Koliha, 2003. Weyl Type Theorems For Bounded Linear Operators, Acta Sci. Math. 69 (1-2): 359-376.

6. Curto, R.E. and Y.M. Han,2003. Weyl's Theorem for Algebraically Paranormal Operators, Integ. Equat. Oper. Th. 47: 307-314.

7. Dowson, H.R., 1973. Spectral Theory of Linear Operators, Academic Press, London.

8. Guggal, B.P., I.H. Jeon and I.H. Kim, 2006. On Weyl's Theorem for quasi-class A operators, J. Korean Math. Soc., 43(4): 899-909.

9. Duggal, B.P. and S.V. Djordjevic, 2004, Generalized Weyl's Theorem for A class of Operators Satisfying A norm Condition, Math. Proc. Royal Irish Acad., 104A(1): 75-81.

10. Duggal, B.P. and S.V. Djordjevic, 2006. Generalized Weyl's Theorem for A class of Operators Satisfying A norm Condition II, Math. Proc. Royal Irish Acad., 104A(1): 1-9.
11. Duggal, B.P., 1997. The Weyl Spectrum of p-hyponormal Operators, Integ. Equat. Oper. Th., 29: 197-201.

12. Duggal, B.P., 2005. Weyl's Theorem for Algebraically Totally Hereditarily Normaloid Operators, J. Math. Anal. Appl., 308: 578-587.

13. Finch, J.K., 1975. The Single Valued Extension Property on a Banach Space, Pacific J. Math., 58 (1): 61-69.

14. Harte, R. and W.Y. Lee, 1997. Another Note On Weyl's Theorem, Trans. Amer. Math. Soc., 349 (5): 2115-2124.

15. Jeon, I.H. and I.H. Kim, 2006. On operators satisfying $\mathrm{T}^{*}\left|\mathrm{~T}^{2}\right| \mathrm{T} \geq \mathrm{T}^{*}|\mathrm{~T}|^{2} \mathrm{~T}$, Linear alg. Appl., 418: 854-862.

16. Koliha, J.J., 1996. Isolated Spectral Points, Proc. Amer. Math. Soc., 124 (11): 3417-3424.

17. Lahrouz, M. and M. Zohry, 2005. Weyl Type Theorems and the Approximate point Spectrum, Irish Math. Soc. Bulletin, 55: 41-51.

18. Laursen, K.B., 1992. Operators with Finite Ascent, Pacific J. Math. 152 (2): 323-336.

19. Laursen, K.B., 1997. Essential Spectra Through Local Spectral Theory, Proc. Amer. Math. Soc., 125 (5): 1425-1434.

20. Rakocevic, V., 1999. Operators obeying a-Weyl's theorem, Publ. Math. Debrecen, 55 (3-4): 283-298.

21. Zguitti H., 2006. A note on Generalized Weyl's Theorem, J. Math. Anal. Appl., 316: 373-381. 